\title{
Supporting Policy Definition In The E-Health Domain: A QCA Based Method
}

Authors:

Paolo Spagnoletti (corresponding author), CeRSI-LUISS Guido Carli University, via Alberoni 7, Roma, Italy - pspagnoletti@luiss.it, phone: +39-06-85225795, fax: +3906-844078510

Valentina Albano, CeRSI-LUISS Guido Carli University, via Alberoni 7, Roma, Italy valbano@luiss.it

Alessandro D’Atri, CeRSI-LUISS Guido Carli University, via Alberoni 7, Roma, Italy-datri@luiss.it

\begin{abstract}
eHealth is broadly considered as a promising strategy to improve the economic sustainability and quality of the healthcare services provision in Europe. Nevertheless, despite the enthusiastic declarations of eHealth potential, the adoption of Information Technology in healthcare has progressed very slowly. A critical factor, not deeply addressed in literature, is related to the process of prioritization of the eHealth solutions to adopt in presence of financial constraints, external and internal pressures from a wide range of heterogeneous stakeholders, and conflicting information on different technological solutions. In this paper we introduce a method supporting policy definition in the eHealth domain. This method is based on a qualitative comparative analysis (QCA) of best practices and previous experiences performed through the lens of an analytic framework whose dimensions and categories are well situated in the eHealth context. This method could support policy-makers in the identification of the properties and characteristics of innovative projects at European level as well as in the analysis of the gap between the international scenario and the local context, in order to understand trends and dynamics of development, to evaluate the best opportunities for innovation and, therefore, to assign priorities for the next investments by respecting the constraints of available resources.
\end{abstract}

Keywords: eHealth policy, Qualitative Comparative Analysis, Set-theoretic method

\section{INTRODUCTION}

The European Commission with the eHealth Action Plan (EC, 2004) and the recent Digital Agenda for Europe (EC, 2010), has assigned to eHealth a pivotal role in the present and future socio-economic and financial challenges faced by national healthcare authorities in Europe. Recent studies show that healthcare systems need to deal with a steady population ageing and the diffusion of chronic diseases that contribute to the increase of demand for health and social services (Pomerleau et al, 2008). At the same time, citizens increase their expectations regarding the access to better information, better expertise, better quality of 
medical services, innovative treatments, safer care and support in long term care and independent living, as well as support in their lifestyle management (EC, 2007). These challenges together with others, such as the growing phenomenon of the cross-border healthcare (due to the patients mobility), are leading to a substantial increase in healthcare expenditure (RAND and Capgemini, 2010). In this scenario, exacerbated by the increasing financial constraints incumbent upon healthcare providers (EC, 2004), the exploitation of the enormous potential of eHealth services and solutions becomes necessary to improve overall healthcare delivery (Pagliari, et al. 2007).

Several efforts in defining the term e-Health and its scope have been provided in the academic environment: in term of personal understanding (Eysenbach, 2001); as systematic review of published definition (Oh et al., 2005); and for future research agenda as a "scoping review" (Pagliari et al., 2005) or through stakeholder consultation and policy context review (Jones et al., 2005). Mitchell (2000), refers to eHealth as an umbrella term, describing the combined use of electronic communication and information technology in the healthcare sector, as well as the use of digital data - transmitted, stored and retrieved electronically - for clinical, educational and administrative purposes, both at the local site and at distance. It is widely believed that e-Health can address many of the problems currently faced by the healthcare systems, improving quality of care, increasing efficiency of healthcare work, assuring healthcare services more accessible and better effectiveness of medical interventions and patient care (Stroetmann et al, 2006). Nevertheless, despite the enthusiastic declarations of eHealth potential, the adoption of information technology has been much slower in healthcare than in other industries such as banking and manufacturing (Simon et al, 2007; Bates, 2005). Cost is often cited as the primary reason of the slow rate of eHealth adoption, followed by the lack of methods to evaluate the effective benefits provided to stakeholders (cost saving, improved patient satisfaction, operating efficiencies, quality of care, and patient safety), and privacy and security concerns (Angst and Agarwal, 2009, Dixon, 2007). Another critical factor, not deeply addressed in literature, is related to the process of prioritization of the eHealth solution to adopt, in presence of financial constraints. Faced with continuous streams of new technological solutions, decision makers have to deal with many external and internal challenges (i.e. external pressure from patients requiring more transparency, internal pressure from physicians and healthcare managers, as well as conflicting information on different technological solutions). This raises the need for the development of decisionmaking methods and tools supporting policy makers dealing with these issues.

In this paper we introduce a method supporting policy definition in the eHealth domain. The method is based on the comparative analysis of good practices and previous experiences performed through the lens of an analytic framework whose dimensions and categories are well situated in the eHealth context. The contribution is organized as follows: in the next Section we provide an overview of the main factors of complexity influencing the definition process of eHealth policies. Then in Section 3 a new method supporting the decision makers will be presented. In Section 4 we provide an example of application of the method, based on data collected from an EU eHealth project database. Suggestions for future research conclude the paper in Section 5.

\section{DECISION MAKING IN THE E-HEALTH DOMAIN}

Making investment decisions in the eHealth domain is a critical task. First, eHealth is an interdisciplinary area. It needs efforts and contributions from areas of trust, ethical, juridical, economic, political, informatics, and methodologies. As a matter of example, Personal Data Protection legislation and standards pose some issues when applied to cross- 
regional interoperability. The successful implementation of eHealth project cannot be achieved without joint efforts from several disciplines (IANIS, 2007). Second, eHealth is now on the governmental agenda of all EU Members States (EC, 2009). This high attention on eHealth investments has created a strong European eHealth market with a very wide range of applications for all needs (Gartner, 2008). It is expected to reach more than EUR 15 million by 2012, with a compounded annual growth rate of $2.9 \%$ (RAND and Capgemini, 2010). Third, the essence of eHealth is that it should facilitate the transforming of healthcare processes for the benefit of patients and the healthcare system (IANIS, 2007). To realize this essence, the decision maker is surrounded by a wide variety of solutions that can support all types of health services: health promotion, diagnosis, therapy, rehabilitation or long-term care. eHealth can also underpin support activities like management and administration, logistics and supply of health-related goods, facilities management as well as public health, continued medical education, or medical research and clinical trials (EC, 2009). The choice among all these ways to improve and change healthcare, depends on the main priorities that have been identified. Both the potential benefits and the needs to be met are many and often eHealth solutions influence a number of these simultaneously (Stroetmann et al., 2006). The priority, for instance, may be to meet the needs of patients/citizens focusing on objectives such as equal access, timeliness of care, safety, quality information, cross-border healthcare, effectiveness of care, empowerment, etc. As an alternative, is it possible to assign priority in supporting the operational processes of healthcare professionals by focusing on objectives such as data sharing among healthcare organizations, cost-cutting strategies, selecting necessary services, addressing the shortages in qualified staff, etc. Finally, in Europe healthcare is either a national or a regional responsibility. In the same way, the use of eHealth applications differs from nation to nation and from region to region (IANIS, 2007). In this context, characterized by different factors, it is difficult to identify best practices which are universally applicable, but only good practices that can be a success under different circumstances (EC, 2009). Furthermore, decision makers could take advantage from the availability of methods for selecting optimal eHealth applications with respect to expected benefits and risks (Rigby, 2006).

In order to deal with these challenges a method to support decision makers in making their choices about eHealth investments by taking into account the above mentioned priorities, benefits, problems and potentials, is under development. This method is based on the qualitative comparative analysis (QCA) of a set of eHealth projects both internationally and locally. The method has practical implications for eHealth decision makers, by supporting them in the identification of the properties and characteristics of innovative projects at European level and to analyze the gap between the international scenario and the local context. In this way it is possible to understand trends and dynamics of development, to evaluate the best opportunities for innovation and, therefore, to assign priorities for the next investments by respecting the constraints of available resources.

\section{THE POLICY DEFINITION METHOD}

The method we propose can support the policy definition process through an analysis of previous eHealth experiences. It is based on the application of a data analytic strategy known as qualitative comparative analysis, or QCA. It refers to the analysis of dichotomous social data reflecting the memberships of cases in conventional, crisp sets. In-depth discussions of this method can be found in Ragin $(1987,2006)$. In order to perform the above mentioned comparative analysis among eHealth initiatives, we base our work on the application of set-theoretic methods for studying cases as configurations. According with Ragin (1987, 2006), set-theoretic methods differ from conventional, variable-based 
approaches in that they do not disaggregate cases into independent, analytically separate aspects but, instead, treat configurations as different types of cases. To examine these different configurations of attributes, set-theoretic methods use Boolean algebra, a notational system that permits the algebraic manipulation of logical statements (Fiss, 2007). This allows simplifying the complexity of causal relationships by reducing them to primitive expressions and formulating more succinct Boolean statements. Moreover, whenever both the number of categories and the number of cases are small, is it useful to display graphically a Boolean data set through Venn diagrams. Such an approach in many ways offers a better fit with a configurational understanding of eHealth initiatives and also allows for a sophisticated assessment of how different causes combine to affect relevant outcomes such as for instance project success.

According with the objectives of this work, we propose the use of set-theoretic methods to examine how different elements characterizing eHealth projects, combine rather than compete to produce an outcome. From the perspective of decision makers, this approach contributes to move beyond simple contingency approaches where either environmental or technology related aspects are considered as a source for making decisions. In fact, also in the eHealth domain, most organizations face multiple contingencies, such as previously adopted strategies and structures, activities, and technologies, with significant interdependencies among these contingencies (Galunic and Eisenhardt, 1994). Furthermore, these multiple contingencies may present contradictory requirements for strategy and structure (Miller, 1992; Fiss, 2007). Moreover, set-theoretic methods allow performing a qualitative comparative analysis when the number of cases is too small for many conventional statistical analyses such as between ten and fifty cases (Fiss, 2007). Thus, it results appropriate for comparing eHealth implementation projects at a local level, such as for instance within a regional area or within a single country.

The first step of our QCA based method consisted in identifying the unit of analysis and then defining the analytical framework based on dimensions and categories (sets) through which cases will be classified. This analytical framework has been defined through a conceptual analysis performed by researchers and domain experts. The next section presents the output of this activity.

\subsection{The analytical framework}

The unit of analysis we considered in our method corresponds to a single eHealth project implemented in a given area. With respect to the QCA method, each project represents a case and a set of classes have been defined in order to allow the researchers to classify cases. A first distinction provided by the taxonomy is related to the "target patients" to be addressed and the "organizational choices" faced by the decision maker. With respect to the "organizational choice" class, the taxonomy provides 34 categories grouped along 4 main dimensions (Table 1).

The first dimension is related to the relationship supported by the ICT system. The categories associated with this dimensions correspond to all the possible pairs (including reflective pairs) of subjects that have been identified: Patient/Citizen; Professional, Administrator, Manager). The second dimension is related to the phase of assistance sociomedical process where the ICT system operates: Prevention, Access, Treatment, Monitoring and control. The third dimension is the type of ICT system implemented. Categories proposed by European Commission (2007, p. 10) have been adopted:

- Clinical Information Systems: a) Specialized tools for health professionals within care institutions (e.g. Radiology or Nursing Information Systems) b) Tools for 
primary care and/or for outside the care institutions (e.g. General Practitioner or Pharmacy Information Systems).

- Secondary Usage Non-Clinical Systems: a) systems for health education and health promotion of patients/citizens (e.g. health portals, online health information services); b) specialized systems for researchers and public health data collection and analysis (e.g. bio statistical programs for infectious diseases, drug development); c) support systems such as supply chain management, billing systems, administrative and management systems.

- Telemedicine: personalized health systems and services (e.g. remote patient monitoring, tele-consultation).

- Integrated Health Clinical Information Network: distributed electronic health record systems and associated services such as e-prescriptions or e-referrals.

The fourth dimension is the level of supported cooperation. The categories associated with this dimensions are:

- Intra-organizational: automation of a single activity or integration of activities within the healthcare process.

- Inter-organizational: collaboration between a) multiple healthcare providers (network); b) between different types of public and private institutions (Public Private Networks); c) exchange of healthcare clinical data between patients and physicians, nurses and other specialists (clinical based patient's participation); d) exchange of non-clinical information such as quality of services, good practices, etc. within a particular community of patients/citizens (Info based patient's participation).

Table 1: The "organizational choices" taxonomy

\begin{tabular}{|c|c|c|}
\hline \multicolumn{3}{|c|}{ Organizational choices } \\
\hline Dimensio & & Categories \\
\hline \multirow{9}{*}{\multicolumn{2}{|c|}{ Relationship supported }} & Patient/C.-Patient/Citizen \\
\hline & & HC Professional-HC Prof. \\
\hline & & Administrator-Admin \\
\hline & & Manager-Manager \\
\hline & & Patient/C.-Professional \\
\hline & & Patient/C.-Administrator \\
\hline & & Professional-Administrator \\
\hline & & Professional-Manager \\
\hline & & Manager-Administrator \\
\hline \multirow{14}{*}{ Phase of socio-medical process } & \multirow{3}{*}{ Prevention } & Sensitisation campaigns \\
\hline & & Management vaccines \\
\hline & & Screening \\
\hline & \multirow{6}{*}{ Access } & Emergency \\
\hline & & Specialist visits \\
\hline & & Hospitalisation \\
\hline & & Drugs-prosthesis \\
\hline & & Social services \\
\hline & & Home care \\
\hline & \multirow{4}{*}{ Treatment } & Diagnosis \\
\hline & & Therapy \\
\hline & & Socio-medical assessment \\
\hline & & Assistance intervention \\
\hline & Monitoring and control & Clinical monitoring data \\
\hline
\end{tabular}




\begin{tabular}{|c|c|c|}
\hline & & Administrative monitoring data \\
\hline \multirow{4}{*}{\multicolumn{2}{|c|}{ Type of ICT system }} & Clinical IS \\
\hline & & $\begin{array}{l}\text { Secondary Usage Non-Clinical } \\
\text { Systems }\end{array}$ \\
\hline & & Telemedicine \\
\hline & & $\begin{array}{l}\text { Integrated Health Clinical } \\
\text { Information Network }\end{array}$ \\
\hline \multirow{6}{*}{ Level of supported cooperation } & \multirow{2}{*}{ Intra-org. } & Automation \\
\hline & & Integration \\
\hline & \multirow{4}{*}{ Inter-org. } & Network \\
\hline & & Public Private Networks \\
\hline & & $\begin{array}{l}\text { Clinical based patient's } \\
\text { participation }\end{array}$ \\
\hline & & Info based patient's participation \\
\hline
\end{tabular}

With respect to the "target patient" class, the categories are listed in Table 2 under three dimensions: Risk categories, Chronic patients, Others.

Table 2: The "target patients" taxonomy

\begin{tabular}{|c|c|}
\hline \multirow{2}{*}{ Dimensions } & Target patients \\
\hline \multirow{3}{*}{ Risk categories } & Categories \\
\cline { 2 - 2 } & Elderly \\
\cline { 2 - 2 } & Maternity \\
\cline { 2 - 2 } Chronic patients & Dental diseases \\
\cline { 2 - 2 } & Amyotrophic lateral sclerosis \\
\cline { 2 - 2 } & Diabetes \\
\cline { 2 - 2 } & Alzheimer \\
\hline \multirow{2}{*}{ Others } & Tumours \\
\cline { 2 - 2 } & Acute cases \\
\hline
\end{tabular}

\subsection{The comparative method}

Once the final objective of the analysis has been defined, researchers will select within the above mentioned taxonomy one outcome variable and a subset of categories to be used as contingency factors of the analysis. Furthermore, a selection of cases (eHealth experiences) which are relevant with respect to investigation goals will be identified and analyzed. This is done by interpreting case data and by filling with 1 and 0 values a matrix where rows correspond to cases and columns correspond to the contingency factors and to the outcome. Such values represent the membership or the non membership of each case to the corresponding category respectively. The truth table obtained through this process, will be further analyzed in order to identify configurations of contingency factors affecting the outcome. This can be done with the support of software packages implementing one of the possible algorithms for the analysis of crisp data sets.

For the purpose of this paper we propose to adopt a software tool for small number analysis which allows the graphical representation of cases on a Venn diagram with up to five independent conditions (Cronqvist, 2005). Our assumption is that by graphically positioning 
"good practice" cases on a map with possible configurations of contingency factors, the decision maker can better identify which policy can lead to the desired outcome.

\section{EXAMPLE OF APPLICATION}

In this section we aim to provide a brief example of application for the above mentioned method, based on the comparative analysis of a set of European initiatives considered "good practices" in eHealth. In order to collect information on the characteristics of these good practices, we refer to a public available online database created in the context of an initiative of the European Commission (Good eHealth, EC 2008). The Good eHealth initiative is a three-year study (from 2006 to 2008) which has been financed under the former Modinis programme in the Directorate-General of Information Society and Media. The objectives of this study are to i) identify good practices and their associated benefits; ii) develop and implement proven approaches to wider dissemination and transfer real-life experiences; iii) stimulate and foster accelerated take-up of e-Health by addressing the common challenges of e-Health and lessons learned. These objectives are in line with the purposes of our example which aims to show how a decision maker can be supported in defining its eHealth policy.

Among the 132 solutions which were listed in the database at the time of data collection, 94 cases were certified as "quality reviewed cases". The project website describes in detail the selection process through which cases have been analyzed by the expert panel. A common template is used for presenting cases on the website in order to provide a minimum amount of information for each good practice. The average size of the overall case description is about 2000 words. For the purposes of this paper, a team of domain experts researchers and consultants - have classified the 94 "quality reviewed cases" using the categories of the above mentioned taxonomy. The outcome of this phase represents the crisp data set that will be further processed. Moreover, the researchers have collected in a separate table some general information about the projects such as the name, the starting date, the country, some comments, and references to further documentation. These additional data are useful to support the selection process of cases by reducing the data set to a small number of cases.

Let now suppose that a decision maker wants to understand how to deal with chronic diseases (such as diabetes, cancer, Alzaimer or cardiovascular disease). These pathologies represent the most common cause of mortality or disability throughout the world (WHO, 2005 ) and are responsible for almost the $70 \%$ of healthcare expenditures (Mongan et al, 2008). In this scenario a comprehensive and integrated action for chronic care management has been defined as "vital investments" (WHO 2005). Among the policy definition issues in the domain of chronic care management, a decision maker must choose in which type of ICT systems to invest and which should be the boundaries of the cooperative environment (e.g. medical department, hospital, territorial Healthcare network...). In fact, assuming that an effective management of chronic conditions requires a coordinated and proactive organization of care involving a multiplicity and variety of players and both clinical and administrative acts, a possible investigation can be related to the type of ICT systems implemented (widely recognized as critical coordination mechanisms) and to the actual level of integration supported (intra-organization vs inter-organization) expressed in the proposed taxonomy under the "level of supported cooperation" dimension. With this aim, by selecting projects with $\mathrm{CHR}=1$ (Chronic patients) from the crisp data set, we obtain a subset of 23 cases. In fact, since we are analyzing good practice cases, it is possible to assume that whenever the CHR value is 1 , the project has effectively achieved the goal of addressing the needs of chronic diseases. 
As explained before, a possible set of elements influencing the capability to address chronic diseases needs are the inter-organizational character of the initiative and the type of ICT systems implemented. The corresponding sets which have been considered in this example are: INT (inter-organizational), CIS (Clinical IS), SUNCS (Secondary Usage NonClinical Systems), TLM (Telemedicine), and IHCIN (Integrated Health Clinical Information Network). These sets can be considered as contingency factors having an impact on the CHR outcome. By using the Quine algorithm on the Tosmana 1.3.1 software package, is it possible to calculate the truth table (Table 3) and to graphically visualize the distribution of cases on a Venn diagram (Figure 1).

Table 3: Truth table

\begin{tabular}{|l|c|c|c|c|c|}
\hline \multicolumn{1}{|c|}{ CASE ID } & INT & CIS & SUNCS & TLM & IHCIN \\
\hline CASE 1,CASE 59,CASE 64,CASE 98 & 0 & 1 & 1 & 0 & 0 \\
\hline CASE 146,CASE 91 & 1 & 1 & 0 & 1 & 1 \\
\hline CASE 69,CASE 46,CASE 47 & 1 & 0 & 1 & 1 & 0 \\
\hline CASE 5,CASE 50,CASE 139 & 1 & 1 & 0 & 1 & 0 \\
\hline CASE 20,CASE 142,CASE 36 & 1 & 0 & 1 & 0 & 1 \\
\hline CASE 68 & 0 & 0 & 1 & 0 & 0 \\
\hline CASE 83 & 1 & 1 & 1 & 1 & 1 \\
\hline CASE 35 & 1 & 0 & 0 & 1 & 0 \\
\hline CASE 41 & 1 & 1 & 1 & 0 & 1 \\
\hline CASE 140 & 0 & 0 & 0 & 1 & 0 \\
\hline
\end{tabular}

Figure 1: Venn diagram 


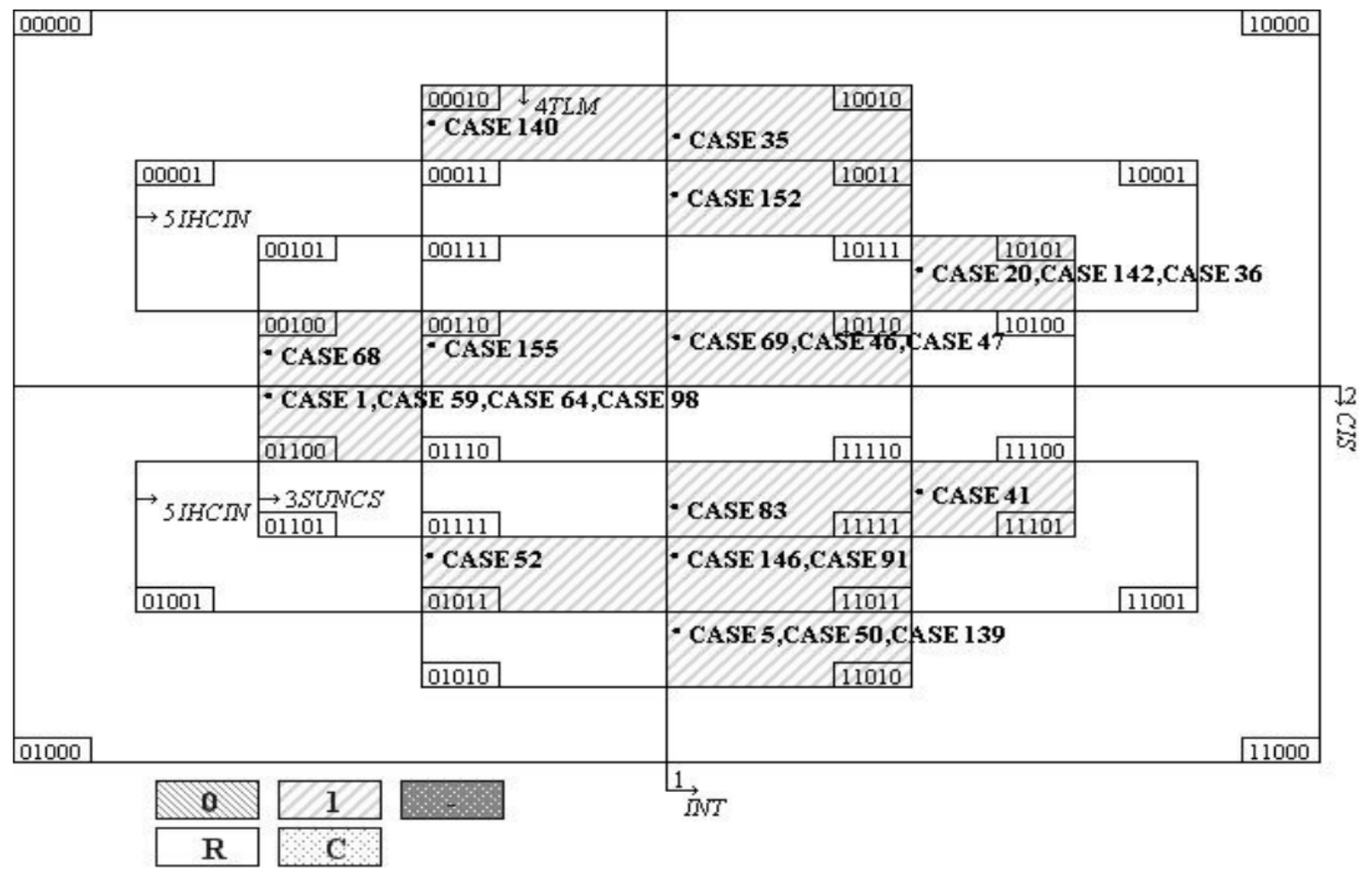

Each area in the diagram represents a possible combination of the selected contingency factors. For instance, area 11111 refers to inter-organizational projects (INT=1) in which all the four categories of ICT systems are implemented (CIS $=1, \mathrm{SUNCS}=1$, TLM=1, IHCIN=1). In our example, case 83 fits with these characteristics and can be deeply analyzed in order to increase the knowledge about hints and issues for these types of projects. It corresponds to the "DITIS: Network for Home HealthCare Collaboration" project, developed in Cyprus between 1999 and 2003 when routine operations started.

According with the project description, "the main purpose of DITIS is to provide continuity of care by supporting the operation of virtual collaborative healthcare teams that care for a single patient at home but do neither normally nor easily come together. Its objectives include: immediate and effective treatment of symptoms based on informed decisions possible through the instant access to the EHR by other care professionals; improved cost effectiveness through effective communication and coordination of healthcare teams and reduction of bureaucratic overhead; improved quality of life for chronic patients and their family". Furthermore, the following Boolean statement represents the minimization of the previous cases through which it is possible to further analyze by the means of multiplecase studies cases represented by each factor:

$\operatorname{INT}\{1\} * \operatorname{SUNCS}\{0\} * \operatorname{TLM}\{1\}+$

CIS $\{0\} * \operatorname{TLM}\{1\} * \operatorname{IHCIN}\{0\}+$

$\operatorname{INT}\{0\} * \operatorname{SUNCS}\{1\} * \operatorname{TLM}\{0\} * \operatorname{IHCIN}\{0\}+$

INT $\{1\} * \operatorname{CIS}\{1\} * \operatorname{TLM}\{1\} * \operatorname{IHCIN}\{1\}+\operatorname{CIS}\{1\} * \operatorname{SUNCS}\{0\} * \operatorname{TLM}\{1\} * \operatorname{IHCIN}\{1\}+$

$\operatorname{INT}\{1\} * \operatorname{SUNCS}\{1\} * \operatorname{TLM}\{0\} * \operatorname{IHCIN}\{1\} \operatorname{INT}\{1\} * \operatorname{SUNCS}\{0\} * \operatorname{TLM}\{1\}+$

CIS $\{0\} *$ TLM $\{1\} * \operatorname{IHCIN}\{0\}+$

$\operatorname{INT}\{0\} * \operatorname{SUNCS}\{1\} * \operatorname{TLM}\{0\} * \operatorname{IHCIN}\{0\}+\operatorname{CIS}\{1\} * \operatorname{SUNCS}\{0\} * \operatorname{TLM}\{1\} * \operatorname{IHCIN}\{1\}+$

$\operatorname{INT}\{1\} * \operatorname{SUNCS}\{1\} * \operatorname{TLM}\{0\} * \operatorname{IHCIN}\{1\}+\operatorname{INT}\{1\}\}^{*} \operatorname{CIS}\{1\}{ }^{*} \operatorname{SUNCS}\{1\}{ }^{*} \operatorname{IHCIN}\{1\}$ 


\section{CONCLUSIONS}

In this paper we have introduced a method supporting policy definition in the eHealth domain. We have also provided an example of application of this method based on empirical data collected from EU sources. The example has shown the potential of the method in supporting a decision maker willing to understand whether to invest in inter or intraorganizational projects and which combinations of ICT systems can be effective. The following steps of the research will consist in the validation of the method involving e-health policy decision makers and in developing new version of the method based on fuzzy logic analysis techniques.

\section{REFERENCES}

Angst C., Agarwal R., (2009) Adoption of Electronic Health Records in the Presence of Privacy Concerns: The Elaboration Likelihood Model and Individual Persuasion, MIS Quarterly 33 (2): 339-370.

Bates, D. W. (2005). Physicians and Ambulatory Electronic Health Records. Health Affairs 24(5):1180-9.

Cronqvist, L. (2005). Tosmana - Tool for small-n analysis. Version 1.201. Marburg: University of Marburg. http://www.tosmana.net/ [cited 2010-10-18].

Dixon B. E. (2007). A roadmap for the adaptation of eHealth. E-Service Journal 5 (3):3-13

Eysenbach, G. (2001). What is e-health?, Journal of Medical Internet Research, 3(2): e20

European Commission (2004). eHealth - making healthcare better for European citizens: An action plan for a European eHealth Area. Brussels, 30 April 2004.

European Commission (2007). eHealth Taskforce report 2007-Accelerating the development of the ehealth market in Europe, Luxembourg: Office for Official Publications of the European Communities.

European Commission (2008) Information Society and Media Directorate-Genaral. Expert Impact Assessment http://kb.good-ehealth.org/search.do

European Commission (2009). Good eHealth Report - eHealth in Action - Good Practice in European Countries, Luxembourg: Office for Official Publications of the European Communities.

European Commission, COM, (2010). European Commission, 2010. A Digital Agenda for Europe, COM, 2010 (245 final).

Fiss, P. (2007) A Set-Theoretic Approach to Organizational Configurations. Academy of Management Review $(50,4)$ 1180-1198

Galunic, D. C., and Eisenhardt, K. M. (1994). Reviewing the strategy-structure-performance paradigm. Research in Organizational Behavior, 16: 215-255.

Gartner, (2008). Hype Cycle for Healthcare Provider Applications and Systems, Stamford.

IANS, (2007). Guide to Regional Good Practice eHealth, Brussels, eris@ -The European Regional Information Society Association.

Jones, R., Rogers, R., et al. (2005). What Is eHealth (5): A Research Agenda for eHealth Through Stakeholder Consultation and Policy Context Review. Journal of Medical Internet Research, 7(5): e54.

Miller, D. (1992). Environmental fit versus internal fit. Organization Science, 3: 159-178.

Mitchell J., (2000). Increasing the cost-effectiveness of telemedicine by embracing e-health. Journal of Telemedine and Telecare, 6:16-19

Mongan, J.J. Ferris, T. G. Lee, T. H. (2008). Options for Slowing the Growth of Health Care Costs. The New England Journal of Medicine, 358(14):1509-1514

Oh, H., Rizo, C., et al. (2005). What Is eHealth (3): A Systematic Review of Published Definitions. Journal of Medical Internet Research, 7(1): e1. 
Pagliari, C., Sloan, D., et al. (2005). What Is eHealth (4): A Scoping Exercise to Map the Field. Journal of Medical Internet Research, 7(1): e9.

Pagliari C., Detmer D., Singleton P. (2007) Potential of electronic personal health records, British Medical Journal 335: 330-333.

Pomerleau, J., Knai C. and Nolte E., (2008). The burden of chronic disease in Europe, in Nolte, E. and McKee, M., (2008), Caring for people with chronic diseases: a health system perspective, Maidenhead, Open University Press, pp. 15-43.

Ragin, C. C. (1987). The comparative method: Moving beyond qualitative and quantitative strategies. Berkeley: University of California Press.

Ragin, C. C. (2006). Set relations in social research: Evaluating their consistency and courage. Political Analysis, 14: 291-310.

RAND Europe and Capgemini Consulting, (2010). Business Models for eHealth, Cambridge: final report prepared for ICT for the Health Unit, DG Information Society and Media European Commission.

Rigby M., (2006). Essential prerequisites to the safe and effective widespread roll-out of eworking in healthcare. International Journal of Medical Informatics 75(2): 138-147.

Simon, S.R., Kaushal, R., Cleary, P.D., Jenter, C.A., Volk, L.A., Poon, E.G., et al. (2007) . "Correlates of Electronic Health Record Adoption in Office Practices: A Statewide Survey" Journal of American Medical Informatics Association 14(1): 110-117

Stroetmann, K.A., Jones, T. Dobrev, A. and Veli, N.O., (2006). eHealth is worth it: the economic benefits of implemented eHealth solutions at ten European sites, Luxembourg: Office for Official Publications of the European Communities, European Commission.

World Health Organization, (2005), Preventing Chronic Disease: a vital investment. WHO Press, Geneva. 